\title{
Why Pursue a Fourth Year in Advanced/Transplant Hepatology?
}

\author{
Arpan Mohanty $^{1} \cdot$ Brett E. Fortune $^{1}$
}

Published online: 12 March 2016

(c) Springer Science+Business Media New York 2016

The traditional pathway for advanced/transplant hepatology training consists of a 1-year fellowship program, accredited by the Accreditation Council for Graduate Medical Education (ACGME), and is usually completed after finishing a 3-year gastroenterology fellowship-thus referred to as a "fourth year." However, in hopes to increase the number of trained transplant hepatologists given the growing job demand, the American Board of Internal Medicine (ABIM) and American Association for the Study of Liver Diseases (AASLD) have developed a collaborative pilot program, which combines training in gastroenterology and advanced/transplant hepatology within a 3-year timeline at selected institutions [1]. So far, 31 fellows (14 fellows for the current academic year 2015-2016) have enrolled in the program. Interval data evaluating the early metrics of the pilot program are not yet available. This article will focus on the potential strengths in pursuing a fourth year of training in advanced/transplant hepatology as well as the potential pitfalls of the combined pilot program.

In the last few decades, immense strides have been made in the discovery, diagnosis, prevention, and management of liver diseases. The incipient field of hepatology continues to evolve with the discovery of novel pathophysiologic mechanisms, drugs, therapeutic modalities, and diagnostic approaches. In spite of this brisk progress, mortality rates from chronic liver disease and liver cancer have increased substantially over the past three decades [2]. Liver cancer is the seventh largest contributor of cancer mortality in the

Brett E. Fortune

brett.fortune@yale.edu

1 Section of Digestive Diseases, Yale University School of Medicine, P.O. Box 208019, New Haven, CT 06520, USA
USA [3]. Even with the availability of highly effective hepatitis $\mathrm{C}$ virus (HCV) therapies, it is estimated that 300,000 persons will die of $\mathrm{HCV}$ in the next three decades [4]. Nonalcoholic fatty liver disease (NAFLD) is estimated to affect $10-46 \%$ of the American population, and the morbidity and mortality associated with NAFLD continue to rise $[5,6]$. Although nearly six thousand people underwent liver transplantation in 2015, nearly 15,000 remain actively listed for liver transplantation today [7]. In the foreseeable future, the practice of hepatology will evolve and become more complex with increased applications of personalized medicine as seen with the use of genomics in management of hepatocellular carcinoma. Thus, with rapid expansion of medical knowledge in the field of hepatology and due to the substantial disease burden from liver diseases, there remains great demand for dedicated professional expertise in hepatology. Yet, there is a shortage of providers to fulfill this need and groups have attempted to create pathways in hope to increase the number of such providers. A search on a popular employment search website, www.indeed.com, shows at least twenty current listings for hepatologists across the country (date of search: February 5, 2016). The three-year ABIM pilot training program in gastroenterology and advanced/transplant hepatology was instituted to address the shortage of trained clinical hepatologists in the coming years. However, the "fourth year" still remains relevant, especially for the training of future physician-scientists, whether in basic or clinical research, as this next generation will be instrumental in the continued advancement in the field of hepatology.

The traditional training pathway in advanced/transplant hepatology provides opportunity for meaningful research and career development with the provision of dedicated research time during the first 3 years of gastroenterology 
training. We are currently witnessing paradigm shifts in diagnosis and management of liver diseases, based on meteoric growth in basic and clinical research with ample academic and industry interest. It is therefore a great time for fellows to immerse in research and better understand the emerging and future trends in hepatology. The dedicated research time during the three-year gastroenterology fellowship can be used to acquire necessary research skills, become familiar with funding mechanisms within different research tracks, and develop strong mentor-mentee relationships and collaborations. In addition, this track allows time for education on process analysis and practice quality improvement that are both critical topics for any posttraining employment. The combined gastroenterology/advanced hepatology fellowship is primarily clinically focused and limits adequate time for scholarly pursuits and participation in quality improvement projects. These limitations could negatively affect the fellows' career development and academic enthusiasm.

Other limitation consists of the early commitment required for the pilot program that potentially pressures fellows into pursuing this track without a chance to explore their personal interests within other aspects of gastroenterology and change their mind regarding their career path. Additionally, fellows participating in the pilot program may have too many clinical commitments and hinder proper patient-physician relationships as well as collaborations with other disciplines, such as working on a transplant team managing pre- and post-transplant patients as well as exposure to rare and complex cases. Alternative strategies to promote career development as well as cultivating academic pursuits for fellows include enrollment onto a NIH T32 training grant during their gastroenterology fellowship at participating programs as well as applying for the AASLD advanced/transplant hepatology fellowship award.

During the academic year 2014-2015, 41 transplant hepatology fellows (31 fourth-year fellows and 10 fellows in the pilot program) were enrolled in 43 ACGME accredited competency-based advanced/transplant hepatology fellowship programs. Data from the pilot program are still being collected, and the success of this program will be assessed on multiple fronts including competence of graduating fellows in gastroenterology and hepatology, board pass rates, and proportion of fellows who remain in the field. Exit surveys from graduating fellows should be used to assess preparedness for real-world jobs. Reverse survey data from employers should also be considered to assess competency of graduating fellows.

As the field of hepatology progresses, training competency and career satisfaction will need to be constantly reassessed. While the combined pilot track may be appealing due to its shorter duration, "the fourth year" remains an exciting route as it provides opportunities for academic exploration and development for intellectual curiosity that in the future will lead to academic success and enhancement in the field. We agree that measures must be taken to increase the training of future hepatologists in order to meet the growing clinical demand, but we must make sure that proper education and experience are allotted for fellows' career development and avoid "shortcuts."

\section{References}

1. Rustgi VK, Davis GL, Herrine SK, et al. Future trends in hepatology: challenges and opportunities. Hepatology. 2008;48: 655-661.

2. Kim Y, Ejaz A, Tayal A, et al. Temporal trends in populationbased death rates associated with chronic liver disease and liver cancer in the United States over the last 30 years. Cancer. 2014; $120: 3058-3065$.

3. Siegel R, Naishadham D, Jemal A. Cancer statistics, 2013. $C A$ Cancer J Clin. 2013;63:11-30.

4. Chhatwal J, Wang X, Kanwal F, et al. Hepatitis C disease burden in the United States in 2015 and beyond. Hepatology. 2015;62:33A-92A.

5. Vernon G, Baranova A, Younossi ZM. Systematic review: the epidemiology and natural history of non-alcoholic fatty liver disease and non-alcoholic steatohepatitis in adults. Aliment Pharmacol Ther. 2011;34:274-285.

6. Williams CD, Stengel J, Asike MI, et al. Prevalence of nonalcoholic fatty liver disease and nonalcoholic steatohepatitis among a largely middle-aged population utilizing ultrasound and liver biopsy: a prospective study. Gastroenterology. 2011;140:124-131.

7. https://www.unos.org/. Accessed February 5, 2016. 